Da Conroy ${ }^{*}$ ) gefunden hat, dafs die Oberflächenfarbe des krystallisirten Jodes eine bläuliche ist, so würde daraus, dem obigen Farbengesetz gemüls, zu folgern sein, dafs die Moleculargrößse des krystallisirten Jods durch die Formel $J_{6}$ resp. $n\left(J_{6}\right)$ auszudrücken sei. Die Moleculargröfse des flüssigen Jods könnte dann $\mathrm{J}_{4}$ resp. $\mathrm{n}\left(\mathrm{J}_{4}\right)$ des gasförmigen Jodes $J_{2}$ resp. $n\left(J_{y}\right) \rightleftharpoons$ sein.

\title{
A n h a n g.
}

Krystallographische Untersuchung einiger Polyjodide; von $O$. Liidecke.

Tetramethylammoniumenneajodid, $\left(\mathrm{CH}_{3}\right)_{6} \mathrm{NJ}_{\mathbf{8}}$.

Schmelzpunkt $110^{\circ}$. Es krystallisirt höchst wahrscheinlich im rhombischen Krystallsystem. Figur 1 stellt einen mikroscopisch gemessenen Krystall dar. Alle
sind tafelförmig nach $\mathrm{c}=\mathrm{OP}$, dem basischen
Pinakoïd; seitlich werden die Tafeln durch
die beiden anderen Pinakoide a $=\infty \ddot{\mathrm{P}}_{\infty}$
und $\mathrm{b}=\infty \ddot{\mathrm{P}} \infty$, sowie durch die Säulen zu c mit der Kante von $b: c$ einen Winkel von $138^{\circ}$ und

*) Procesd. of Royal Society, London. Vol. Ð5, 51. 
mit der von $c$ : $S$ einen Winkel von $160^{\circ}$ bildet. An anderen Krystallen fanden sich zum Theil andere Säulen zwischen a und $b$, doch war die rhombische Symmetrie immer gewahrt. Die Hexima der Auslöschung liegen in c parallel den Kanten zu a und b. Stellt man im Orthoscop bei entferntem Analysator den Krystall mil der Kante a : b parallel dem Huuptschnitt des Polarisators, so erscheint der Krystall gelblichroth; hingegen ist der parallel b : c in c schwingende Strahl fast schwarz. Im auffallenden Licht sehen die Krystalle beinahe metallglänzend grünlich-schwarz aus. Die angeführten Messungen wurden unter einem Leitz'schen Mikroscop ausgeführt.

Tetruäthylammoniumheptajodid, $\left(\mathrm{C}_{8} \mathrm{H}_{8}\right)_{4} \mathrm{NJ}_{7}$.

Schmelzpunkt $108^{\circ}$. Es bildet schwarzbraune rhombische Krystalle, wie dieselben in Figur 2 dargestellt sind. Bei $\left\{\begin{array}{l}\text { grofser Dünne werden sie mit brauer Farbe durch- } \\ \text { sichtig, sie erweisen sich dann als nicht pleochroï- } \\ \text { tisch. Betrachtet man } \mathrm{c}=\mathrm{OP} \text { als basisches Pina- } \\ \text { koïd der rhombischen Krystalle, so ist a }=\infty \overline{\mathrm{P}} \infty \\ \text { das Makropinakoïd und } \mathrm{b}=\infty \mathrm{P} \infty \text { das Brachy- }\end{array}\right.$ pinakoïd; an den Krystallen finden sich dann noch Flächen zwischen a und $b$, welche einer Säule $m=\infty \mathrm{P}$ angchören; dieselbe bildet nit a einen Winkel von 136 bis $138^{\circ}$. Die Maxima der Auslöschungen liegen in c den Kanten zu a und b parallel. Die Krystalle sind gewolnnlich zum Theil hohl und so zerbrechlich, dafs sie sich nicht auf Wachs aufspiefsen lassen; ein dickerer resistenzfähiger Krystall ergab auf dem Babinet'schen Reflexionsgoniometer a : b $=8^{\circ} 55^{\prime} ; \mathrm{b}: \mathrm{a}_{1}$ $=90^{\circ} 0^{\prime} ; a^{\prime}: b^{\prime}=89^{\circ} 55^{\prime}$. Die Säulenflächen waren an diesem Krystall nicht entwickelt und solche mit $m$ gelang es nicht aufzuspiefsen, ohne dafs sie zerbrachen. 
Phenyltrimethylammoniumtrijodid,

$$
\left(\mathrm{C}_{6} \mathrm{H}_{3}\right)\left(\mathrm{CH}_{8}\right)_{3} \mathrm{NJ}_{3} \text {. }
$$

Schmelzpunkt 1150. Es bildet rhombische braune tafeligo Krystalle. In Figur 3 ist ein solcher Krystall in einer Projection auf die Basis $c=0 P$ darge-

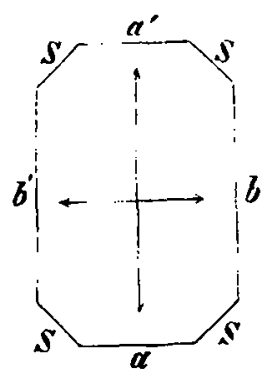
stellt. Seitlich werden dieselben ron sehr schmalen, linienartig entwickelten Flächen begrenzt und zwar von $=$ $\infty \check{\mathrm{P}} \infty, \mathrm{b}=\infty \overline{\mathrm{P}} \infty$ und $\mathrm{S}=\infty \mathrm{P}$. Die Sãule $S$ macht mit a einen Winkel von $140^{\circ}$ und mit $b$ einen solchen von 130. Der in c parallel b schwingende Strahl erscheint hellgelb, während der parallel a schwingende rothbraun ist. Die Maxima der Auslöschung gehen in $c$ parallel $a$ und $b$.

Phenyltrimethylammoniumpentajodid, $\left(\mathrm{C}_{6} \mathrm{H}_{6}\right)\left(\mathrm{CH}_{3}\right)_{5} \mathrm{NJ}_{5}$.

Die Vermuthung von Schorschmidt (Wien. Ac. Ber. 1883) bestätigt sich : die Krystalle dieses Körpers sind monoklin. Das Axenverhältnifs ist :

$$
\text { a }: \mathrm{b}: \mathrm{c}=1,7943: 1: 1,2511 \text {, bei } \beta=69^{\circ} 43^{\prime} \text {. }
$$

Die Krystalle zeigen die Combinationen $\mathrm{a}=\infty \mathrm{P} \infty 100$, $\mathrm{b}=\infty \mathrm{P} \infty 01 \mathrm{C}, \mathrm{c}=\mathrm{OP} 001, \mathrm{~m}=\infty \mathrm{P} 110, \mathrm{n}=\infty \mathrm{P}^{\prime 2}$ $210, \mathrm{~d}=+2 P^{2} \infty$ 201. Es wurde gemessen :
L ïdocke gemessen berechnet
Bchorschmidt
$\mathrm{a}: \mathrm{c}=\mathrm{i} 00: 00 \mathrm{i}=110^{\circ} 17^{\prime}$
a : $m=100: 110=120^{\circ} 43^{\prime}$

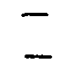
a : $\mathrm{a}=100: 210=139^{\circ} 45^{\prime} \mathrm{ca}$
$139^{\circ} 55,1^{\prime}$
$\mathrm{d}: \mathrm{c}=20 \overline{\mathrm{i}}: 00 \overline{\mathrm{i}}=111^{\circ} 33^{\prime}$
c: $\mathrm{m}=001: 110=100^{\circ} 35^{\prime}$
$100^{\circ} 12^{\prime}$

Interessant sind die optischen Eigenschaften der Krystalle; in sehr dünnen Blättchen sind sie gelblich durchsichtig und 
zeigen starken Pleochroismus : in der Fläche $\mathrm{c}=001$ parallel der Axe b dunkelbraun und parallel der Axe a gelblichroth. Dickere Krystalle zeigen den senkrecht zur Axe b schwingenden Strahl braunroth, den parallel dazu schwingenden vollständig absorbirt, schwarz. Bei Tagesbeleuchtung sind die Krystalle käfergrün mit einem Stich in's metallisch Gelbe; sieht man in der Richtung der Axe b auf die abgebrochenen Krystalle, so erscheinen sie tief blau. Im Lampenlicht erscheinen sie grünlich-schwarz. Die beiden vorhergehend erwähnten Farbenerscheinungen dürften daher mit der Fluorescenz im Zusammenhang stehen.

\section{Phenyltrimethylammoniumheptajodid, $\left(\mathrm{C}_{8} \mathrm{H}_{6}\right)\left(\mathrm{CH}_{8}\right)_{8} \mathrm{NJ}_{7}$.}

Es krystallisirt wahrscheinlich im monoklinen System. Figur 4 stellt einen Krystall dieser Verbindung dar. Sie sind

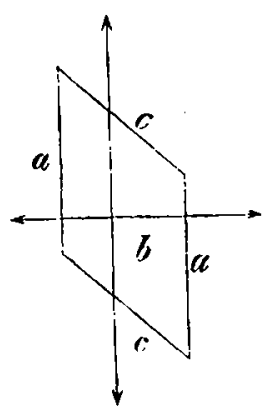
alle tafelig nach der als Symmetrieebene aufgefafsten Fläche $b=\infty \mathrm{P} \infty$. Die seitlichen Flächen $c$ sind dann $O P$ und $a=\infty l^{2} \infty$. Der Winkel von $a: c$ beträgt $61^{\circ}$. Die Maxima der Auslöschungen liegen parallel und senkrecht zu a. Der parallel a in c schwingende Strahl wird von den Krystallen vollständig absorbirt; der senkrecht dazu in derselben Fläche schwingende wird von sehr dünnen Blättchen (unter $1 / 80 \mathrm{~mm}$ ) gelb durchgelassen, von dickeren rothbraun. Noch dickere Krystalle sind undurchsichtig und erscheinen im auffallenden Licht chlorit-grün. Auf dem Goniometer liefsen sich die Krystalle leider nicht messen. 
Phenyldimethyläthylammoniumtrijodid, $\left(\mathrm{C}_{6} \mathrm{H}_{8}\right)\left(\mathrm{CH}_{8}\right)_{8}\left(\mathrm{C}_{8} \mathrm{H}_{5}\right) \mathrm{NJ}_{3}$.

Die Krystalle sind hexagonal rhomboëdrisch mit dem Rhomboëder. Winkel von $72^{\circ} 48^{\prime}$; die auf dem Babinetschen Goniometer vorgenommenen Messungen der 3 Polkanten schwanken an demselben Krystall von $72^{\circ} 42^{\prime}$ bis $72^{\circ} 54^{\prime}$. Die Auslöschungen liegen parallel den Diagonalen der Rhomboëderflächen.

$$
\begin{aligned}
& \text { Phenyldimethyläthylammoniumpentajodid, } \\
& \qquad\left(\mathrm{C}_{6} \mathrm{H}_{6}\right)\left(\mathrm{CH}_{9}\right)_{2}\left(\mathrm{C}_{8} \mathrm{H}_{5}\right) \mathrm{NJ}_{6} .
\end{aligned}
$$

Das Salz stellt sich in dunkelgrünen chloritähnlichen Blättchen dar. Bei sehr grofser Dünne der Blättchen sind dieselben braun durchsichtig, wenn langgestreckte Krystalle mit ihrer Haupterstreckung parallel dem Hauptschnitt des Polarisators liegen; in senkrechter Richtung sind sie dunkler mit einem gelblich-grünen Schiller.

Die Maxima der Auslöschungen liegen theils parallel der Längserstreckung, theils bilden dieselben kleine Winkel damit; da anderweitige Flächen nicht vorhanden waren, so liefs sich das Krystallsystem auch nicht annähernd vermuthen (monoklin oder triklin?).

$$
\begin{aligned}
& \text { Phenyldimethyläthylammoniumheptajodid, } \\
& \qquad\left(\mathrm{C}_{6} \mathrm{H}_{5}\right)\left(\mathrm{CH}_{8}\right)_{8}\left(\mathrm{C}_{2} \mathrm{H}_{5}\right) \mathrm{NJ}_{7} .
\end{aligned}
$$

Es bildet Krystalle von schwarzblauer Farbe und zeigt keine scharfen ausgebildeten Kanten, obwohl einzelne glänzende Flächen imıner sichtbar sind. Der parallel der Haupterstreckung schwingende Strahl wird fast vollständig durch die Krystalle absorbirt, der senkrecht dazu schwingende wird mit brauner Farbe durchgelassen; anderes Weiteres ist nicht bestimmbar. 


\section{Trimethyläthylammoniumpentajodid,}

$$
\left(\mathrm{CH}_{8}\right)_{8}\left(\mathrm{C}_{9} \mathrm{H}_{5}\right) \mathrm{NJ}_{5} \text {. }
$$

Schmelzpunkt $26^{\circ}$. Es scheint rhombisch zu sein; vielleicht kann man die Krystalle als rhombische Combinationen von $\mathbf{c}=0 \mathrm{P}, \mathrm{a}=\infty \mathrm{P}^{\mathrm{P}} \infty, \mathrm{b}=\infty \check{\mathrm{P}} \infty, \mathrm{m}=\infty \mathrm{P}$ und $\mathrm{d}$ $=\breve{\mathrm{P}}_{\infty}$ auffassen; sie sind tafelförmig nach c. Messen lassen sich die Krystalle nicht.

$$
\begin{aligned}
& \text { Trimethyläthylammoniumtrijodid, } \\
& \qquad\left(\mathrm{CH}_{3}\right)_{3}\left(\mathrm{C}_{3} \mathrm{H}_{5}\right) \mathrm{NJ}_{3} .
\end{aligned}
$$

Es soll nach Müller rhombisch krystallisiren.

Die Krystalle sind kleine Würfelchen, welche sich im Orthoscop und Konoscop als vollkommen isotrop erweisen. Auf dem Babinet'schen Reflexionsgoniometer wurde gemessen $001: 100=89^{\circ} 59,5^{\prime}$ und $100: 010=90^{\circ} 14^{\prime}$ ca ; der dritte Winkel 001 : 010 wurde mit Hülfe des Mikroscops zu ca. $90^{\circ}$ bestimmt. Die Kanten des $W$ ürfels werden regelmärsig abgestumpf durch sehr schmale Flächen des halbflächig ausgebildeten Pyramidenwürfels $\propto 03$ (310): es wurde gemessen :

$010: 310=161^{\circ} 33^{\prime}$; die Theorie verlangt $161^{\circ 3} 34^{\prime}$.

Die Krystalle sind also regulär und zwar parallelfächig hemiëdrisch.

$$
\begin{gathered}
\text { Triäthylmethylammoniumtrijodid, } \\
\left(\mathrm{C}_{9} \mathrm{H}_{5}\right)_{8} \mathrm{CH}_{3} \mathrm{NJ}_{8} .
\end{gathered}
$$

Es soll nach Müller quadratisch krystallisiren.

Die Krystalle lassen eine genauere Bestimmung auf dem Goniometer nicht zu, da die Endflächen niemals ausgebildet sind; es sind rectanguläre Blättchen, in welchen die Maxima der Auslöschung parallel den Uinrandungen liegen; ziemlich lebhaften Pleochroïsmus besitzen sie : der parallel der Hauptausdehnungsrichtung schwingende Strahl wird gelb, der senkrecht $\mathrm{zu}$ diesem schwingende roth durchgelassen. 
Teträ̈thylammoniumtrijodid, $\left(\mathrm{C}_{8} \mathrm{H}_{5}\right)_{4} \mathrm{NJ}_{3}$.

Nach Haidinger's Messungen ) tetragonal mit dem Axenverhältnifs a : $\mathrm{c}=1: 0,671$ und den Flächen $0=P$, $\mathrm{d}^{2}=, \mathrm{p}=\infty \mathrm{P}, \mathrm{a}=\infty \mathrm{P} \infty$ und $\mathrm{c}=0 \mathrm{P}$. Die von Geuther dargestellten sind Combinationen, theils von $P$ und $\infty \mathrm{P}, \infty \mathrm{P} \infty$ (mikroscopisch beobachtet), theils von $0=\mathrm{P}$, $c=0 \mathrm{P}$ und $\mathrm{p}=\infty \mathrm{P}$ (auf dem Goniometer gemessen).

\begin{tabular}{|c|c|c|c|c|}
\hline \multirow[b]{2}{*}{ 1) $0: 0=111: 111$} & \multicolumn{2}{|c|}{$\begin{array}{c}\text { Haidinger } \\
\text { berechnet }\end{array}$} & \multicolumn{2}{|c|}{$\underset{\text { Hamessen }}{\text { Haidinger }} \underset{\text { ud }}{\operatorname{cock} \theta}$} \\
\hline & $=$ & $121^{\circ} 46^{\prime}$ & $121^{0} 44^{\prime}$ & - \\
\hline $0: 0=111: 11 i$ & $=$ & - & $86^{05} 9^{\prime *}$ & $87^{\circ} 18^{\prime}$ \\
\hline $0: p=111: 110$ & $=$ & $133^{\circ} 30^{\prime}$ & - & $133^{\circ} 36^{\prime}$ \\
\hline $11 \overline{1}: 110$ & $=$ & $133^{\circ} 30^{\circ}$ & - & $133^{\circ} 28^{\prime}$ \\
\hline $0: c=111: 001$ & $=$ & $136^{0} 30^{\prime}$ & - & $136^{\circ} 27^{\prime}$ \\
\hline iī : 001 & $=$ & $136^{\circ} 30^{\prime}$ & - & $136^{\circ} 16^{\prime}$ \\
\hline 2) $0: 0=i i 1: 1 \mathrm{i} 1$ & $=$ & $58^{\circ} 14^{\prime}$ & - & $60^{0} 3^{\prime}$ \\
\hline $11 \overline{1}: 1 \overline{11}$ & $=$ & $121^{\circ} 46^{\prime}$ & - & $119^{\circ} 57^{\prime}$. \\
\hline
\end{tabular}

Die Bilder der Flächen sind nicht sehr vollkommen, doch zeigen die Messungen, dals die $\mathrm{H}$ a id ing er 'schen Dimensionen auch für diese Krystalle angenommen werden können. Die Maxima der Auslöschungen der roth durchscheinenden Krystalle liegen in $\infty P$ parallel der Hauptaxe. Die Krystalle besitzen lebhaften Dichroismus. Der parallel der Hauptaxe schwingende Strahl wird dunkel rothbraun, der parallel der Nebenaxe schwingende gelblichroth durchgelassen. Sie sind lebhaft diamantglänzend; der bläuliche Schein, welchen die sonst rothbraunen Krystalle im auffallenden Licht zeigen, dürfte mit der Fluorescenz in Verbindung zu bringen sein, da derselbe im Lampenlicht sich bedeutend minderl, ja fast ganz verschwindet.

*) Diese Anualen 1 1, 34. 
Tetramethylammoniumpentajodid,

$$
\left(\mathrm{CH}_{3}\right)_{\&} \mathrm{NJ}_{5} \text {. }
$$

Nach Rainmelsberg und Schabus sind es niedrige monokline Prismen der Combination : $0=-\mathrm{P}(111) ; 0^{\prime}=$ $P(111), p=\infty P(110), b=\infty P \infty(010), c=0 P(001)$. Hinzukommt an den vorliegenden Krystallen $\mathrm{a}=\infty \mathrm{P}^{\mathrm{P}} \infty$ (100); dieselben sind lang säulenförmig nach $\infty \mathrm{P}$ und an dem oberen Ende ist nur $c=0 \mathrm{P}$ ausgebildet. Das von Schabus angenommene Axensystem lautet : $\beta=72^{020,5}$ uud $a: b: c$ $=0,9866: 0,6553$.

\begin{tabular}{|c|c|c|c|c|}
\hline & Berechnot & & Gomesser & \\
\hline & Schabus & Schabus & $\operatorname{Rammelsb}$ & Lüdecke \\
\hline$p=110: 1 \overline{10}=$ & - & $93^{0} 32^{\prime}$ & $94^{\circ} 0^{\prime}$ & $93^{\circ} 42^{\prime}$ \\
\hline$: c=110: 001=$ & - & $102^{\circ} 46^{\prime}$ & $103^{\circ} 40^{\prime}$ & $102^{\circ} 23^{\prime}$ \\
\hline $0 \quad c=111: 001=$ & - & $143^{\circ} 30^{\prime}$ & $143^{\circ} 12^{\prime}$ & - \\
\hline$p: p=110: \overline{1} 10=$ & $86^{\circ} 28^{\prime}$ & - & - & $86^{\circ} 15^{\prime}$ \\
\hline$: p=\overline{100}: \overline{1} \overline{1}_{0}=$ & $=136^{\circ} 46^{\prime}$ & 一 & - & $137^{\circ} 0^{\prime}$ \\
\hline$: p=010: 110=$ & $=133^{\circ} 14^{\prime}$ & - & - & $133^{\circ} 14^{\prime}$. \\
\hline
\end{tabular}

3) Ueber den Bitterstoff der Kalmuswurzel; von $A$. Geuther.

Im vorigen Jahre erschien eine von der philosophischen Facultät in Erlangen approbirte Doctordissertation : den Bitterstoff der Kalmuswurzel" von Herm. Thoms*), welche sich auf Versuche stützt, die Hr. Thoms, früher Assistent in dem unter der Direction des Herrn Prof. Dr.

*) Die Dissertation ist abgedruckt im Archiv I'barm. [3] 94, 465. 